\title{
Solving Relational MDPs with Exogenous Events and Additive Rewards
}

\author{
Saket Joshi ${ }^{1}$, Roni Khardon ${ }^{2}$, Prasad Tadepalli ${ }^{3}$, Aswin Raghavan ${ }^{3}$, and Alan Fern ${ }^{3}$ \\ 1 Cycorp Inc., Austin, TX, USA \\ 2 Tufts University, Medford, MA, USA \\ ${ }^{3}$ Oregon State University, Corvallis, OR, USA
}

\begin{abstract}
We formalize a simple but natural subclass of service domains for relational planning problems with object-centered, independent exogenous events and additive rewards capturing, for example, problems in inventory control. Focusing on this subclass, we present a new symbolic planning algorithm which is the first algorithm that has explicit performance guarantees for relational MDPs with exogenous events. In particular, under some technical conditions, our planning algorithm provides a monotonic lower bound on the optimal value function. To support this algorithm we present novel evaluation and reduction techniques for generalized first order decision diagrams, a knowledge representation for realvalued functions over relational world states. Our planning algorithm uses a set of focus states, which serves as a training set, to simplify and approximate the symbolic solution, and can thus be seen to perform learning for planning. A preliminary experimental evaluation demonstrates the validity of our approach.
\end{abstract}

\section{Introduction}

Relational Markov Decision Processes (RMDPs) offer an attractive formalism to study both reinforcement learning and probabilistic planning in relational domains. However, most work on RMDPs has focused on planning and learning when the only transitions in the world are a result of the agent's actions. We are interested in a class of problems modeled as service domains, where the world is affected by exogenous service requests in addition to the agent's actions. In this paper we use the inventory control (IC) domain as a motivating running example and for experimental validation. The domain models a retail company faced with the task of maintaining the inventory in its shops to meet consumer demand. Exogenous events (service requests) correspond to arrival of customers at shops and, at any point in time, any number of service requests can occur independently of each other and independently of the agent's action. Although we focus on IC, independent exogenous service requests are common in many other problems, for example, in fire and emergency response, air traffic control, and service centers such as taxicab companies, hospitals, and restaurants. Exogenous events present a challenge for planning and reinforcement learning algorithms because the number of possible next states, the "stochastic branching factor", grows exponentially in the number of possible simultaneous service requests.

In this paper we consider symbolic dynamic programming (SDP) to solve RMDPs, as it allows to reason more abstractly than what is typical in forward planning and reinforcement learning. The SDP solutions for propositional MDPs can be adapted to 
RMDPs by grounding the RMDP for each size to get a propositional encoding, and then using a "factored approach" to solve the resulting planning problem, e.g., using algebraic decision diagrams (ADDs) [5] or linear function approximation [4]. This approach can easily model exogenous events [2] but it plans for a fixed domain size and requires increased time and space due to the grounding. The relational (first order logic) SDP approach [3] provides a solution which is independent of the domain size, i.e., it holds for any problem instance. On the other hand, exogenous events make the first order formulation much more complex. To our knowledge, the only work to have approached this is [17|15]. While Sanner's work is very ambitious in that it attempted to solve a very general class of problems, the solution used linear function approximation, approximate policy iteration, and some heuristic logical simplification steps to demonstrate that some problems can be solved and it is not clear when the combination of ideas in that work is applicable, both in terms of the algorithmic approximations and in terms of the symbolic simplification algorithms.

In this paper we make a different compromise by constraining the class of problems and aiming for a complete symbolic solution. In particular, we introduce the class of service domains, that have a simple form of independent object-focused exogenous events, so that the transition in each step can be modeled as first taking the agent's action, and then following a sequence of "exogenous actions" in any order. We then investigate a relational SDP approach to solve such problems. The main contribution of this paper is a new symbolic algorithm that is proved to provide a lower bound approximation on the true value function for service domains under certain technical assumptions. While the assumptions are somewhat strong, they allow us to provide the first complete analysis of relational SDP with exogenous events which is important for understanding such problems. In addition, while the assumptions are needed for the analysis, they are not needed for the algorithm that can be applied in more general settings. Our second main contribution provides algorithmic support to implement this algorithm using the GFODD representation of [8]. GFODDs provide a scheme for capturing and manipulating functions over relational structures. Previous work has analyzed some theoretical properties of this representation but did not provide practical algorithms. In this paper we develop a model evaluation algorithm for GFODDs inspired by variable elimination (VE), and a model checking reduction for GFODDs. These are crucial for efficient realization of the new approximate SDP algorithm. We illustrate the new algorithm in two variants of the IC domain, where one satisfies our assumptions and the other does not. Our results demonstrate that the new algorithm can be implemented efficiently, that its size-independent solution scales much better than propositional approaches [5]19], and that it produces high quality policies.

\section{Preliminaries: Relational Symbolic Dynamic Programming}

We assume familiarity with basic notions of Markov Decision Processes (MDPs) and First Order Logic [14[13]. Briefly, a MDP is given by a set of states $S$, actions $A$, transition function $\operatorname{Pr}\left(s^{\prime} \mid s, a\right)$, immediate reward function $R(s)$ and discount factor $\gamma<1$. The solution of a MDP is a policy that maximizes the expected discounted total reward obtained by following that policy starting from any state. The Value Iteration algorithm 
(VI), calculates the optimal value function $V^{*}$ by iteratively performing Bellman backups $V_{i+1}=T\left[V_{i}\right]$ defined for each state $s$ as,

$$
V_{i+1}(s) \leftarrow \max _{a}\left\{R(s)+\gamma \sum_{s^{\prime}} \operatorname{Pr}\left(s^{\prime} \mid s, a\right) V_{i}\left(s^{\prime}\right)\right\}
$$

Relational MDPs: Relational MDPs are simply MDPs where the states and actions are described in a function-free first order logical language. In particular, the language allows a set of logical constants, a set of logical variables, a set of predicates (each with its associated arity), but no functions of arity greater than 0 . A state corresponds to an interpretation in first order logic (we focus on finite interpretations) which specifies (1) a finite set of $n$ domain elements also known as objects, (2) a mapping of constants to domain elements, and (3) the truth values of all the predicates over tuples of domain elements of appropriate size (to match the arity of the predicate). Atoms are predicates applied to appropriate tuples of arguments. An atom is said to be ground when all its arguments are constants or domain elements. For example, using this notation empty $\left(x_{1}\right)$ is an atom and empty(shop23) is a ground atom involving the predicate empty and object shop 23 (expressing that the shop shop 23 is empty in the IC domain). Our notation does not distinguish constants and variables as this will be clear from the context. One of the advantages of relational SDP algorithms, including the one in this paper, is that the number of objects $n$ is not known or used at planning time and the resulting policies generalize across domain sizes.

The state transitions induced by agent actions are modeled exactly as in previous SDP work [3]. The agent has a set of action types $\{A\}$ each parametrized with a tuple of objects to yield an action template $A(x)$ and a concrete ground action $A(o)$ (e.g. template $\operatorname{unload}(t, s)$ and concrete action unload(truck1, shop2)). To simplify notation, we use $x$ to refer to a single variable or a tuple of variables of the appropriate arity. Each agent action has a finite number of action variants $A_{j}(x)$ (e.g., action success vs. action failure), and when the user performs $A(x)$ in state $s$ one of the variants is chosen randomly using the state-dependent action choice distribution $\operatorname{Pr}\left(A_{j}(x) \mid A(x)\right)$.

Similar to previous work we model the reward as some additive function over the domain. To avoid some technical complications, we use average instead of sum in the reward function; this yields the same result up to a multiplicative factor.

Relational Expressions and GFODDs: To implement planning algorithms for relational MDPs we require a symbolic representation of functions to compactly describe the rewards, transitions, and eventually value functions. In this paper we use the GFODD representation of [8] but the same ideas work for any representation that can express open-expressions and closed expressions over interpretations (states). An expression represents a function mapping interpretations to real values. An open expression $f(x)$, similar to an open formula in first order logic, can be evaluated in interpretation $I$ once we substitute the variables $x$ with concrete objects in $I$. A closed expression (aggregate $f(x)$ ), much like a closed first order logic formula, aggregates the value of $f(x)$ over all possible substitutions of $x$ to objects in $I$. First order logic limits $f(x)$ to have values in $\{0,1\}$ (i.e., evaluate to false or true) and provides the aggregation max (corresponding to existential quantification) and min (corresponding to universal quantification) that can be used individually on each variable in $x$. 
Expressions are more general allowing for additional aggregation functions (for example, average) so that aggregation generalizes quantification in logic, and allowing $f(x)$ to take numerical values. On the other hand, our expressions require aggregation operators to be at the front of the formulas and thus correspond to logical expressions in prenex normal form. This enables us to treat the aggregation portion and formula portion separately in our algorithms. In this paper we focus on average and max aggregation. For example, in the IC domain we might use the expression: " $\max _{t}, \operatorname{avg}_{s}$, (if $\neg \operatorname{empty}(s)$ then 1 , else if $\operatorname{tin}(t, s)$ then 0.1 , else 0$)$ ). Intuitively, this awards a 1 for any non-empty shop and at most one shop is awarded a 0.1 if there is a truck at that shop. The value of this expression is given by picking one $t$ which maximizes the average over $s$.

GFODDs provide a graphical representation and associated algorithms to represent open and closed expressions. A GFODD is given by an aggregation function, exactly as in the expressions, and a labeled directed acyclic graph that represents the open formula portion of the expression. Each leaf in the GFODD is labeled with a non-negative numerical value, and each internal node is labeled with a first-order atom (allowing for equality atoms) where we allow atoms to use constants or variables as arguments. As in propositional diagrams [1], for efficiency reasons, the order over nodes in the diagram must conform to a fixed ordering over node labels, which are first order atoms in our case. Figure 1(a) shows an example GFODD capturing the expression given in the previous paragraph.

Given a diagram $B=\left(\right.$ aggregate $\left._{x} f(x)\right)$, an interpretation $I$, and a substitution of variables in $x$ to objects in $I$, one can traverse a path to a leaf which gives the value for that substitution. The values of all substitutions are aggregated exactly as in expressions. In particular, let the variables as ordered in the aggregation function be $x_{1}, \ldots, x_{n}$. To calculate the final value, $\operatorname{map}_{B}(I)$, the semantics prescribes that we enumerate all substitutions of variables $\left\{x_{i}\right\}$ to objects in $I$ and then perform the aggregation over the variables, going from $x_{n}$ to $x_{1}$. We can therefore think of the aggregation as if it organizes the substitutions into blocks (with fixed value to the first $k-1$ variables and all values for the $k$ 'th variable), and then aggregates the value of each block separately, repeating this from $x_{n}$ to $x_{1}$. We call the algorithm that follows this definition directly brute force evaluation. A detailed example is shown in Figure 3 (a). To evaluate the diagram in Figure 3 a) on the interpretation shown there we enumerate all $3^{3}=27$ substitutions of 3 objects to 3 variables, obtain a value for each, and then aggregate the values. In the block where $x_{1}=a, x_{2}=b$, and $x_{3}$ varies over $a, b, c$ we get the values $3,2,2$ and an aggregated value of $7 / 3$. This can be done for every block, and then we can aggregate over substitutions of $x_{2}$ and $x_{1}$. The final value in this case is $7 / 3$.

Any binary operation op over real values can be generalized to open and closed expressions in a natural way. If $f_{1}$ and $f_{2}$ are two closed expressions, $f_{1}$ op $f_{2}$ represents the function which maps each interpretation $w$ to $f_{1}(w)$ op $f_{2}(w)$. We follow the general convention of using $\oplus$ and $\otimes$ to denote + and $\times$ respectively when they are applied to expressions. This provides a definition but not an implementation of binary operations over expressions. The work in [8] showed that if the binary operation is safe, i.e., it distributes with respect to all aggregation operators, then there is a simple algorithm (the Apply procedure) implementing the binary operation over expressions. For example $\oplus$ 


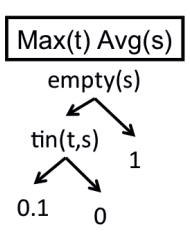

(a)

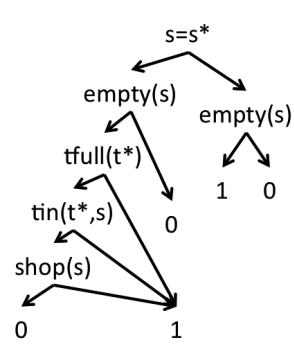

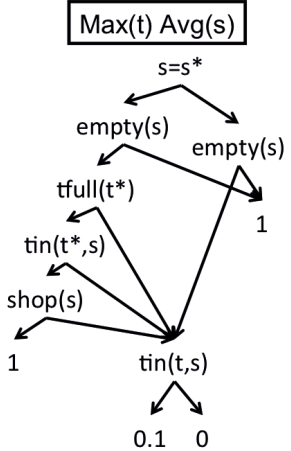

(c)

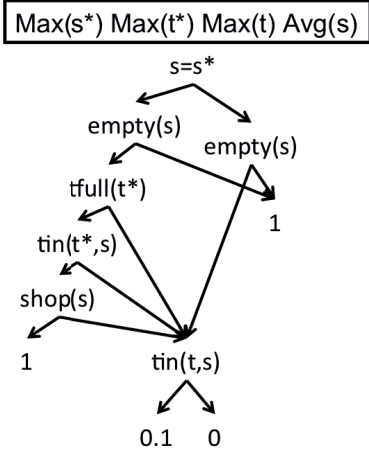

(d)

Fig. 1. IC Dynamics and Regression (a) An example GFODD. (b) TVD for empty(s) under the deterministic action unload $\left(t^{*}, s^{*}\right)$. (c) Regressing the GFODD of (a) over unload $\left(t^{*}, s^{*}\right)$. (d) Object Maximization. In these diagrams and throughout the paper, left-going edges represent the true branch out of the node and right-going edges represent the false branch.

is safe w.r.t. max aggregation, and it is easy to see that $\left(\max _{x} f(x)\right) \oplus\left(\max _{x} g(x)\right)=$ $\max _{x} \max _{y} f(x)+g(y)$, and the open formula portion (diagram portion) of the result can be calculated directly from the open expressions $f(x)$ and $g(y)$. The Apply procedure [208] calculates a diagram representing $f(x)+g(y)$ using operations over the graphs representing $f(x)$ and $g(y)$. Note that we need to standardize apart, as in the renaming of $g(x)$ to $g(y)$ for such operations.

SDP for Relational MDPs: SDP provides a symbolic implementation of the value iteration update of Eq (1) that avoids state enumeration implicit in that equation. The SDP algorithm of [8] generalizing [3] calculates one iteration of value iteration as follows. As input we get (as GFODDs) closed expressions $V_{n}, R$ (we use Figure 1 a) as the reward in the example below), and open expressions for the probabilistic choice of actions $\operatorname{Pr}\left(A_{j}(x) \mid A(x)\right)$ and for the dynamics of deterministic action variants.

The action dynamics are specified by providing a diagram (called truth value diagram or TVD) for each variant $A_{j}(x)$ and predicate template $p(y)$. The corresponding TVD, $T\left(A_{j}(x), p(y)\right)$, is an open expression that specifies the truth value of $p(y)$ in the next state when $A_{j}(x)$ has been executed in the current state. Figure 1 (b) shows the TVD of unload $\left(t^{*}, s^{*}\right)$ for predicates $\operatorname{empty}(s)$. Note that in contrast to other representations of planning operators (but similar to the successor state axioms of [3]) TVDs specify the truth value after the action and not the change in truth value. Since unload is deterministic we have only one variant and $\operatorname{Pr}\left(A_{j}(x) \mid A(x)\right)=1$. We illustrate probabilistic actions in the next section. Following [20]8] we require that $\operatorname{Pr}\left(A_{j}(x) \mid A(x)\right)$ and $T\left(A_{j}(x), p(y)\right)$ have no aggregations and cannot introduce new variables, that is, the first refers to $x$ only and the second to $x$ and $y$ but no other variables. This implies that the regression and product terms in the algorithm below do not change the aggregation function and therefore enables the analysis of the algorithm. 
The SDP algorithm of [8] implements Eq (1) using the following 4 steps. We denote this as $V_{i+1}=S D P^{1}\left(V_{i}\right)$.

1. Regression: The $n$ step-to-go value function $V_{n}$ is regressed over every deterministic variant $A_{j}(x)$ of every action $A(x)$ to produce $\operatorname{Regr}\left(V_{n}, A_{j}(x)\right)$. Regression is conceptually similar to goal regression in deterministic planning but it needs to be done for all (potentially exponential number of) paths in the diagram, each of which can be thought of as a goal in the planning context. This can be done efficiently by replacing every atom in the open formula portion of $V_{n}$ (a node in the GFODD representation) by its corresponding TVD without changing the aggregation function.

Figure 1(c) illustrates the process of block replacement for the diagram of part (a). Note that $\operatorname{tin}()$ is not affected by the action. Therefore its TVDs simply repeats the predicate value, and the corresponding node is unchanged by block replacement. Therefore, in this example, we are effectively replacing only one node with its TVD. The TVD leaf valued 1 is connected to the left child (true branch) of the node and the 0 leaf is connected to the right child (false branch). To maintain the diagrams sorted we must in fact use a different implementation than block replacement; the implementation does not affect the constructions or proofs in the paper and we therefore refer the reader to [20] for the details.

2. Add Action Variants: The Q-function $Q_{V_{n}}^{A(x)}=R \oplus\left[\gamma \otimes \oplus_{j}\left(\operatorname{Pr}\left(A_{j}(x)\right) \otimes\right.\right.$ $\left.\left.\operatorname{Regr}\left(V_{n}, A_{j}(x)\right)\right)\right]$ for each action $A(x)$ is generated by combining regressed diagrams using the binary operations $\oplus$ and $\otimes$ over expressions.

Recall that probability diagrams do not refer to additional variables. The multiplication can therefore be done directly on the open formulas without changing the aggregation function. As argued by [20], to guarantee correctness, both summation steps ( $\oplus_{j}$ and $R \oplus$ steps) must standardize apart the functions before adding them.

3. Object Maximization: Maximize over the action parameters $Q_{V_{n}}^{A(x)}$ to produce $Q_{V_{n}}^{A}$ for each action $A(x)$, thus obtaining the value achievable by the best ground instantiation of $A(x)$ in each state. This step is implemented by converting action parameters $x$ in $Q_{V_{n}}^{A(x)}$ to variables, each associated with the max aggregation operator, and appending these operators to the head of the aggregation function.

For example, if object maximization were applied to the diagram of Figure1(c) (we skipped some intermediate steps) then $t *, s *$ would be replaced with variables and given max aggregation so that the aggregation is as shown in part (d) of the figure. Therefore, in step $2, t *, s *$ are constants (temporarily added to the logical language) referring to concrete objects in the world, and in step 3 we turn them into variables and specify the aggregation function for them.

4. Maximize over Actions: The $n+1$ step-to-go value function $V_{n+1}=\max _{A} Q_{V_{n}}^{A}$, is generated by combining the diagrams using the binary operation max over expressions.

The main advantage of this approach is that the regression operation, and the binary operations over expressions $\oplus, \otimes$, max can be performed symbolically and therefore the final value function output by the algorithm is a closed expression in the same language. We therefore get a completely symbolic form of value iteration. Several instantiations 
of this idea have been implemented [116 6 18 20]. Except for the work of [8 18] previous work has handled only max aggregation. Previous work [8] relies on the fact that the binary operations $\oplus, \otimes$, and max are safe with respect to max, min aggregation to provide a GFODD based SDP algorithm for problems where the reward function has max and min aggregations. In this paper we use reward functions with max and avg aggregation. The binary operations $\oplus$ and $\otimes$ are safe with respect to avg but the binary operation max is not. For example $2+\operatorname{avg}\{1,2,3\}=\operatorname{avg}\{2+1,2+2,2+3\}$ but $\max \{2, \operatorname{avg}\{1,2,3\}\} \neq \operatorname{avg}\{\max \{2,1\}, \max \{2,2\}, \max \{2,3\}\}$. To address this issue we introduce a new implementation for this case in the next section.

\section{Model and Algorithms for Service Domains}

We now proceed to describe our extensions to SDP to handle exogenous events. Exogenous events refer to spontaneous changes to the state without agent action. Our main modeling assumption, denoted A1, is that we have object-centered exogenous actions that are automatically taken in every time step. In particular, for every object $i$ in the domain we have action $E(i)$ that acts on object $i$ and the conditions and effects of $\{E(i)\}$ are such that they are mutually non-interfering: given any state $s$, all the actions $\{E(i)\}$ are applied simultaneously, and this is equivalent to their sequential application in any order. We use the same GFODD action representation described in the previous section to capture the dynamics of $E(i)$.

Example: IC Domain. We use a simple version of the inventory control domain (IC) as a running example, and for some of the experimental results. In IC the objects are a depot, a truck and a number of shops. A shop can be empty or full, i.e., the inventory has only two levels and the truck can either be at the depot or at a shop. The reward is the fraction (average) of non-empty shops. Agent actions are deterministic and they capture stock replacement. In particular, a shop can be filled by unloading inventory from the truck in one step. The truck can be loaded in a depot and driven from any location (shop or depot) to any location in one step. The exogenous action $E(i)$ has two variants; the success variant $E_{\text {succ }}(i)$ (customer arrives at shop $i$, and if non-empty the inventory becomes empty) occurs with probability 0.4 and the fail variant $E_{\text {fail }}(i)$ (no customer, no changes to state) occurs with probability 0.6. Figure 2 parts (a)-(d) illustrate the model for IC and its GFODD representation. In order to facilitate the presentation of algorithmic steps, Figure 2 (e) shows a slightly different reward function (continuing previous examples) that is used as the reward in our running example.

For our analysis we make two further modeling assumptions. A2: we assume that exogenous action $E(i)$ can only affect unary properties of the object $i$. To simplify the presentation we consider a single such predicate $s p(i)$ that may be affected, but any number of such predicates can be handled. In IC, the special predicate $s p(i)$ is empty $(i)$ specifying whether the shop is empty. A3: we assume that $\operatorname{sp}()$ does not appear in the precondition of any agent action. It follows that $E(i)$ only affects $s p(i)$ and that $s p(i)$ can appear in the precondition of $E(i)$ but cannot appear in the precondition of any other action. 


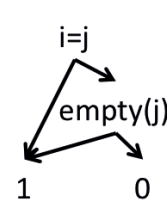

(a)

1

(c)

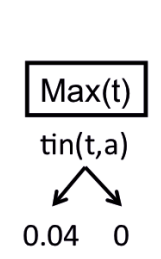

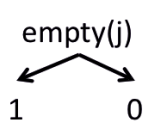

(b)

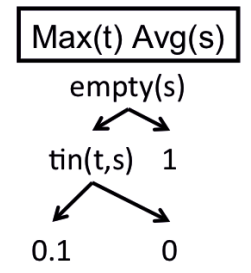

(e)

(d)

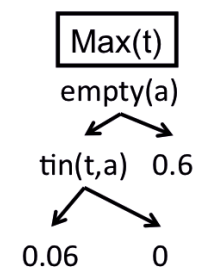

(h)

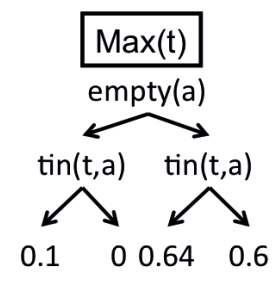

(i)

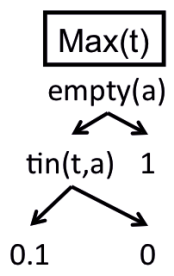

(f)

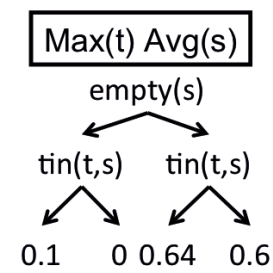

(j)

Fig. 2. Representation and template method for IC. (a) TVD for empty $(j)$ under action variant $E_{\text {succ }}(i)$. (b) TVD for empty $(j)$ under action variant $E_{\text {fail }}(i)$. (c) A specialized form of (a) under $i=j$. This is simply the value 1 and is therefore a GFODD given by a single leaf node. (d) $\operatorname{Pr}\left(E_{\text {succ }}(i) \mid E(i)\right)$ which is simply the value 0.4. (e) A simple reward function. (f) Grounding (e) using Skolem constant $a$. (g) Regressing (f) over $E_{\text {succ }}(a)$ and multiplying with the probability diagram in (d). (h) Regressing (f) over $E_{\text {fail }}(a)$ and multiplying by its probability diagram. (i) Adding (g) and (h) without standardizing apart. (j) Reintroducing the Avg aggregation.

\subsection{The Template Method}

Extending SDP to handle exogenous events is complicated because the events depend on the objects in the domain and on their number and exact solutions can result in complex expressions that require counting formulas over the domain [17 15]. A possible simple approach would explicitly calculate the composition of the agent's actions with all the exogenous events. But this assumes that we know the number of objects $n$ (and thus does not generalize) and results in an exponential number of action variants, which makes it infeasible. A second simple approach would be to directly modify the SDP algorithm so that it sequentially regresses the value function over each of the ground exogenous actions before performing the regression over the agent actions, which is correct by our assumptions. However, this approach, too, requires us to know $n$ and because it effectively grounds the solution it suffers in terms of generality.

We next describe the template method, one of our main contributions, which provides a completely abstract approximate SDP solution for the exogenous event model. We make our final assumption, A4, that the reward function (and inductively $V_{i}$ ) is a closed expression of the form $\max _{x} \operatorname{avg}_{y} V(x, y)$ where $x$ is a (potentially empty) set of variables and $y$ is a single variable, and in $V(x, y)$ the predicate $s p()$ appears instantiated only as $s p(y)$. The IC domain as described above satisfies all our assumptions. 
The template method first runs the following 4 steps, denoted $S D P^{2}\left(V_{i}\right)$, and then follows with the 4 steps of SDP as given above for user actions. The final output of our approximate Bellman backup, $T^{\prime}$, is $V_{i+1}=T^{\prime}\left(V_{i}\right)=S D P^{1}\left(S D P^{2}\left(V_{i}\right)\right)$.

1. Grounding: Let $a$ be a Skolem constant not in $V_{i}$. Partially ground $V$ to get $V=$ $\max _{x} V(x, a)$

2. Regression: The function $V$ is regressed over every deterministic variant $E_{j}(a)$ of the exogenous action centered at $a$ to produce $\operatorname{Regr}\left(V, E_{j}(a)\right)$.

3. Add Action Variants: The value function $V=\oplus_{j}\left(\operatorname{Pr}\left(E_{j}(a)\right) \otimes \operatorname{Regr}\left(V, E_{j}(a)\right)\right)$ is updated. As in $S D P^{1}$, multiplication is done directly on the open formulas without changing the aggregation function. Importantly, in contrast with $S D P^{1}$, here we do not standardize apart the functions when performing $\oplus_{j}$. This leads to an approximation.

4. Lifting: Let the output of the previous step be $V=\max _{x} W(x, a)$. Return $V=$ $\max _{x} \operatorname{avg}_{y} W(x, y)$.

Thus, the algorithm grounds $V$ using a generic object for exogenous actions, it then performs regression for a single generic exogenous action, and then reintroduces the aggregation. Figure 2 parts (e)-(j) illustrate this process.

We now show that our algorithm provides a monotonic lower bound on the value function. The crucial step is the analysis of $S D P^{2}\left(V_{i}\right)$. We have:

Lemma 1. Under assumptions A1, A2, A4 the value function calculated by $S D P^{2}\left(V_{i}\right)$ is a lower bound on the value of regression of $V_{i}$ through all exogenous actions.

Due to space constraints the complete proof is omitted and we only provide a sketch. This proof and other omitted details can be found in the full version of this paper [10].

Proof. (sketch) The main idea in the proof is to show that, under our assumptions, the result of our algorithm is equivalent to sequential regression of all exogenous actions, where in each step the action variants are not standardized apart.

Recall that the input value function $V_{i}$ has the form $V=\max _{x} \operatorname{avg}_{y} V(x, y)=$ $\max _{x} \frac{1}{n}[V(x, 1)+V(x, 2)+\ldots+V(x, n)]$. To establish this relationship we show that after the sequential algorithm regresses $E(1), \ldots, E(k)$ the intermediate value function has the form $\max _{x} \frac{1}{n}[W(x, 1)+W(x, 2)+\ldots+W(x, k)+V(x, k+1)+\ldots+V(x, n)]$. That is, the first $k$ portions change in the same structural manner into a diagram $W$ and the remaining portions retain their original form $V$. In addition, $W(x, \ell)$ is the result of regressing $V(x, \ell)$ through $E(\ell)$ which is the same form as calculated by step 3 of the template method. Therefore, when all $E(\ell)$ have been regressed, the result is $V=\max _{x} \operatorname{avg}_{y} W(x, y)$ which is the same as the result of the template method.

The sequential algorithm is correct by definition when standardizing apart but yields a lower bound when not standardizing apart. This is true because for any functions $f^{1}$ and $f^{2}$ we have $\left[\max _{x_{1}} \operatorname{avg}_{y_{1}} f^{1}\left(x_{1}, y_{1}\right)\right]+\left[\max _{x_{2}} \operatorname{avg}_{y_{2}} f^{2}\left(x_{2}, y_{2}\right)\right] \geq \max _{x}\left[\operatorname{avg}_{y_{1}}\right.$ $\left.f^{1}\left(x, y_{1}\right)+\operatorname{avg}_{y_{2}} f^{2}\left(x, y_{2}\right)\right]=\max _{x} \operatorname{avg}_{y}\left[\left(f^{1}(x, y)+f^{2}(x, y)\right)\right]$ where the last equality holds because $y_{1}$ and $y_{2}$ range over the same set of objects. Therefore, if $f^{1}$ and $f^{2}$ are the results of regression for different variants from step 2, adding them without standardizing apart as in the last equation yields a lower bound.

The lemma requires that $V_{i}$ used as input satisfies A4. If this holds for the reward function, and if $S D P^{1}$ maintains this property then $\mathbf{A 4}$ holds inductively for all $V_{i}$. 
Put together this implies that the template method provides a lower bound on the true Bellman backup. It therefore remains to show how $S D P^{1}$ can be implemented for $\max _{x} \operatorname{avg}_{y}$ aggregation and that it maintains the form $\mathbf{A 4}$.

First consider regression. If assumption $\mathbf{A 3}$ holds, then our algorithm using regression through TVDs does not introduce new occurrences of $\operatorname{sp}()$ into $V$. Regression also does not change the aggregation function. Similarly, the probability diagrams do not introduce $s p()$ and do not change the aggregation function. Therefore A4 is maintained by these steps. For the other steps we need to discuss the binary operations $\oplus$ and max.

For $\oplus$, using the same argument as above, we see that $\left[\max _{x_{1}} \operatorname{avg}_{y_{1}} f^{1}\left(x_{1}, y_{1}\right)\right]+$ $\left[\max _{x_{2}} \operatorname{avg}_{y_{2}} f^{2}\left(x_{2}, y_{2}\right)\right]=\max _{x_{1}} \max _{x_{2}}\left[\operatorname{avg}_{y} f^{1}\left(x_{1}, y\right)+f^{2}\left(x_{2}, y\right)\right]$ and therefore it suffices to standardize apart the $x$ portion but $y$ can be left intact and A4 is maintained.

Finally, recall that we need a new implementation for the binary operation max with avg aggregation. This can be done as follows: to perform $\max \left\{\left[\max _{x_{1}} \operatorname{avg}_{y_{1}}\right.\right.$ $\left.\left.f^{1}\left(x_{1}, y_{1}\right)\right],\left[\max _{x_{2}} \operatorname{avg}_{y_{2}} f^{2}\left(x_{2}, y_{2}\right)\right]\right\}$ we can introduce two new variables $z_{1}, z_{2}$ and write the expression: " $\max _{z_{1}, z_{2}} \max _{x_{1}} \max _{x_{2}} \operatorname{avg}_{y_{1}} \operatorname{avg}_{y_{2}}$ (if $z_{1}=z_{2}$ then $f^{1}\left(x_{1}, y_{1}\right)$ else $f^{2}\left(x_{2}, y_{2}\right)$ )". This is clearly correct whenever the interpretation has at least two objects because $z_{1}, z_{2}$ are unconstrained. Now, because the branches of the if statement are mutually exclusive, this expression can be further simplified to " $\max _{z_{1}, z_{2}} \max _{x} \operatorname{avg}_{y}$ (if $z_{1}=z_{2}$ then $f^{1}(x, y)$ else $f^{2}(x, y)$ )". The implementation uses an equality node at the root with label $z_{1}=z_{2}$, and hangs $f^{1}$ and $f^{2}$ at the true and false branches. Crucially it does not need to standardize apart the representation of $f^{1}$ and $f^{2}$ and thus A4 is maintained. This establishes that the approximation returned by our algorithm, $T^{\prime}\left[V_{i}\right]$, is a lower bound of the true Bellman backup $T\left[V_{i}\right]$.

An additional argument (details available in [10]) shows that this is a monotonic lower bound, that is, for all $i$ we have $T\left[V_{i}\right] \geq V_{i}$ where $T[V]$ is the true Bellman backup. It is well known (e.g., [12]) that if this holds then the value of the greedy policy w.r.t. $V_{i}$ is at least $V_{i}$ (this follows from the monotonicity of the policy update operator $T_{\pi}$ ). The significance is, therefore, that $V_{i}$ provides an immediate certificate on the quality of the resulting greedy policy. Recall that $T^{\prime}[V]$ is our approximate backup, $V_{0}=R$ and $V_{i+1}=T^{\prime}\left[V_{i}\right]$. We have:

Theorem 1. When assumptions A1, A2, A3, A4 hold and the reward function is nonnegative we have for all $i$ : $V_{i} \leq V_{i+1}=T^{\prime}\left[V_{i}\right] \leq T\left[V_{i}\right] \leq V^{*}$.

As mentioned above, although the assumptions are required for our analysis, the algorithm can be applied more widely. Assumptions A1 and A4 provide our basic modeling assumption per object centered exogenous events and additive rewards. It is easy to generalize the algorithm to have events and rewards based on object tuples instead of single objects. Similarly, while the proof fails when A2 (exogenous events only affect special unary predicates) is violated the algorithm can be applied directly without modification. When A3 does not hold, $s p()$ can appear with multiple arguments and the algorithm needs to be modified. Our implementation introduces an additional approximation and at iteration boundary we unify all the arguments of $s p()$ with the average variable $y$. In this way the algorithm can be applied inductively for all $i$. These extensions of the algorithm are demonstrated in our experiments. 
Relation to Straight Line Plans: The template method provides symbolic way to calculate a lower bound on the value function. It is interesting to consider what kind of lower bound this provides. Recall that the straight line plan approximation (see e.g., discussion in [2]) does not calculate a policy and instead at any state it seeks the best linear plan with highest expected reward. As the next observation argues (proof available in [10]) the template method provides a related approximation. We note, however, that unlike previous work on straight line plans our computation is done symbolically and calculates the approximation for all start states simultaneously.

Observation 1. The template method provides an approximation that is related to the value of the best straight line plan. When there is only one deterministic agent action template we get exactly the value of the straight line plan. Otherwise, the approximation is bounded between the value of the straight line plan and the optimal value.

\section{Evaluation and Reduction of GFODDs}

The symbolic operations in the SDP algorithm yield diagrams that are redundant in the sense that portions of them can be removed without changing the values they compute. Recently, [8] introduced the idea of model checking reductions to compress such diagrams. The basic idea is simple. Given a set of "focus states" $S$, we evaluate the diagram on every interpretation in $S$. Any portion of the diagram that does not "contribute" to the final value in any of the interpretations is removed. The result is a diagram which is exact on the focus states, but may be approximate on other states. We refer the reader to [8]7] for further motivation and justification. In that work, several variants of this idea have been analyzed formally (for max and min aggregation), have been shown to perform well empirically (for max aggregation), and methods for generating $S$ via random walks have been developed. In this section we develop the second contribution of the paper, providing an efficient realization of this idea for $\max _{x} \operatorname{avg}_{y}$ aggregation.

The basic reduction algorithm, which we refer to below as brute force model checking for GFODDs, is: (1) Evaluate the diagram on each example in our focus set $S$ marking all edges that actively participate in generating the final value returned for that example. Because we have $\max _{x} \operatorname{avg}_{y}$ this value is given by the "winner" of max aggregation. This is a block of substitutions that includes one assignment to $x$ and all possible assignments to $y$. For each such block collect the set of edges traversed by any of the substitutions in the block. When picking the max block, also collect the edges traversed by that block, breaking ties by lexicographic ordering over edge sets. (2) Take the union of marked edges over all examples, connecting any edge not in this set to 0 .

Consider again the example of evaluation in Figure 3 (a), where we assigned node identifiers $1,2,3$. We identify edges by their parent node and its branch so that the leftgoing edge from the root is edge $1 t$. In this case the final value $7 / 3$ is achieved by multiple blocks of substitutions, and two distinct sets of edges $1 t 2 f 3 t 3 f$ and $1 f 3 t 3 f$. Assuming $1<2<3$ and $f<t, 1 f 3 t 3 f$ is lexicographically smaller and is chosen as the marked set. This process is illustrated in the tables of Figure 3 a). Referring to the reduction procedure, if our focus set $S$ includes only this interpretation, then the edges $1 t, 2 t, 2 f$ will be redirected to the value 0 . 


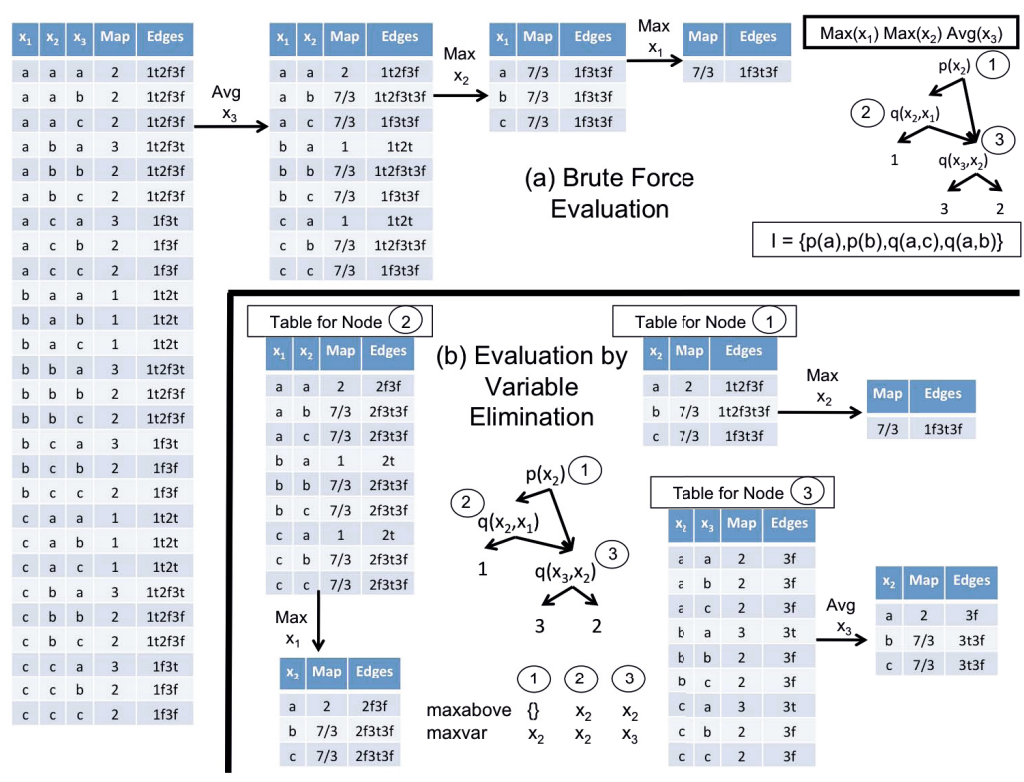

Fig. 3. GFODD Evaluation (a) Brute Force method. (b) Variable Elimination Method.

Efficient Model Evaluation and Reduction: We now show that the same process of evaluation and reduction can be implemented more efficiently. The idea, taking inspiration from variable elimination, is that we can aggregate some values early while calculating the tables. However, our problem is more complex than standard variable elimination and we require a recursive computation over the diagram.

For every node $n$ let $n$.lit $=p(x)$ be the literal at the node and let $n_{\downarrow f}$ and $n_{\downarrow t}$ be its false and true branches respectively. Define $\operatorname{above}(n)$ to be the set of variables appearing above $n$ and sel $f(n)$ to be the variables in $x$. Let maxabove $(n)$ and maxsel $f(n)$ be the variables of largest index in above $(n)$ and self $(n)$ respectively. Finally let maxvar $(n)$ be the maximum between maxabove $(n)$ and maxsel $f(n)$. Figure 3 (b) shows maxvar $(n)$ and maxabove $(n)$ for our example diagram. Given interpretation $I$, let $b l^{n_{\downarrow t}}(I)$ be the set of bindings $a$ of objects from $I$ to variables in $x$ such that $p(a) \in I$. Similarly $b l^{n_{\downarrow f}}(I)$ is the set of bindings $a$ such that $\neg p(a) \in I$. The two sets are obviously disjoint and together cover all bindings for $x$. For example, for the root node in the diagram of Figure $3 \mathrm{~b}$ ), $b l^{n_{\downarrow t}}(I)$ is a table mapping $x_{2}$ to $a, b$ and $b l^{n_{\downarrow f}}(I)$ is a table mapping $x_{2}$ to $c$. The evaluation procedure, $\operatorname{Eval}(n)$, is as follows:

1. If $n$ is a leaf:

(1) Build a "table" with all variables implicit, and with the value of $n$.

(2) Aggregate over all variables from the last variable down to maxabove $(n)+1$.

(3) Return the resulting table.

2. Otherwise $n$ is an internal node:

(1) Let $M^{\downarrow t}(I)=b l^{n_{\downarrow t}}(I) \times \operatorname{Eval}\left(n_{\downarrow t}\right)$, where $\times$ is the join of the tables. 
(2) Aggregate over all the variables in $M^{\downarrow t}(I)$ from the last variable not yet aggregated down to maxvar $(n)+1$.

(3) Let $M^{\downarrow f}(I)=b l^{n_{\downarrow f}}(I) \times \operatorname{Eval}\left(n_{\downarrow f}\right)$

(4) Aggregate over all the variables in $M^{\downarrow f}(I)$ from the last variable not yet aggregated down to maxvar $(n)+1$.

(5) Let $M=M^{\downarrow t}(I) \cup M^{\downarrow f}(I)$.

(6) Aggregate over all the variables in $M$ from the last variable not yet aggregated down to maxabove $(n)+1$.

(7) Return node table $M$.

We note several improvements for this algorithm and its application for reductions, all of which are applicable and used in our experiments. (I1) We implement the above recursive code using dynamic programming to avoid redundant calls. (I2) When an aggregation operator is idempotent, i.e., $\operatorname{op}\{a, \ldots, a\}=a$, aggregation over implicit variables does not change the table, and the implementation is simplified. This holds for max and avg aggregation. (I3) In the case of $\max _{x} \operatorname{avg}_{y}$ aggregation the procedure is made more efficient (and closer to variable elimination where variable order is flexible) by noting that, within the set of variables $x$, aggregation can be done in any order. Therefore, once $y$ has been aggregated, any variable that does not appear above node $n$ can be aggregated at $n$. (I4) The recursive algorithm can be extended to collect edge sets for winning blocks by associating them with table entries. Leaf nodes have empty edge sets. The join step at each node adds the corresponding edge (for true or false child) for each entry. Finally, when aggregating an average variable we take the union of edges, and when aggregating a max variable we take the edges corresponding to the winning value, breaking ties in favor of the lexicographically smaller set of edges.

A detailed example of the algorithm is given in Figure $3 \mathrm{~b}$ ) where the evaluation is on the same interpretation as in part (a). We see that node 3 first collects a table over $x_{2}, x_{3}$ and that, because $x_{3}$ is not used above, it already aggregates $x_{3}$. The join step for node 2 uses entries $(b, a)$ and $(c, a)$ for $\left(x_{1}, x_{2}\right)$ from the left child and other entries from the right child. Node 2 collects the entries and (using I3) aggregates $x_{1}$ even though $x_{2}$ appears above. Node 1 then similarly collects and combines the tables and aggregates $x_{2}$. The next theorem is proved by induction over the structure of the GFODD (details available in [10]).

Theorem 2. The value and max block returned by the modified Eval procedure are identical to the ones returned by the brute force method.

\section{Experimental Validation}

In this section we present an empirical demonstration of our algorithms. To that end we implemented our algorithms in Prolog as an extension of the FODD-PLANNER [9], and compared it to SPUDD [5] and MADCAP [19] that take advantage of propositionally factored state spaces, and implement VI using propositional algebraic decision diagrams (ADD) and affine ADDs respectively. For SPUDD and MADCAP, the domains were specified in the Relational Domain Description Language (RDDL) and translated into 

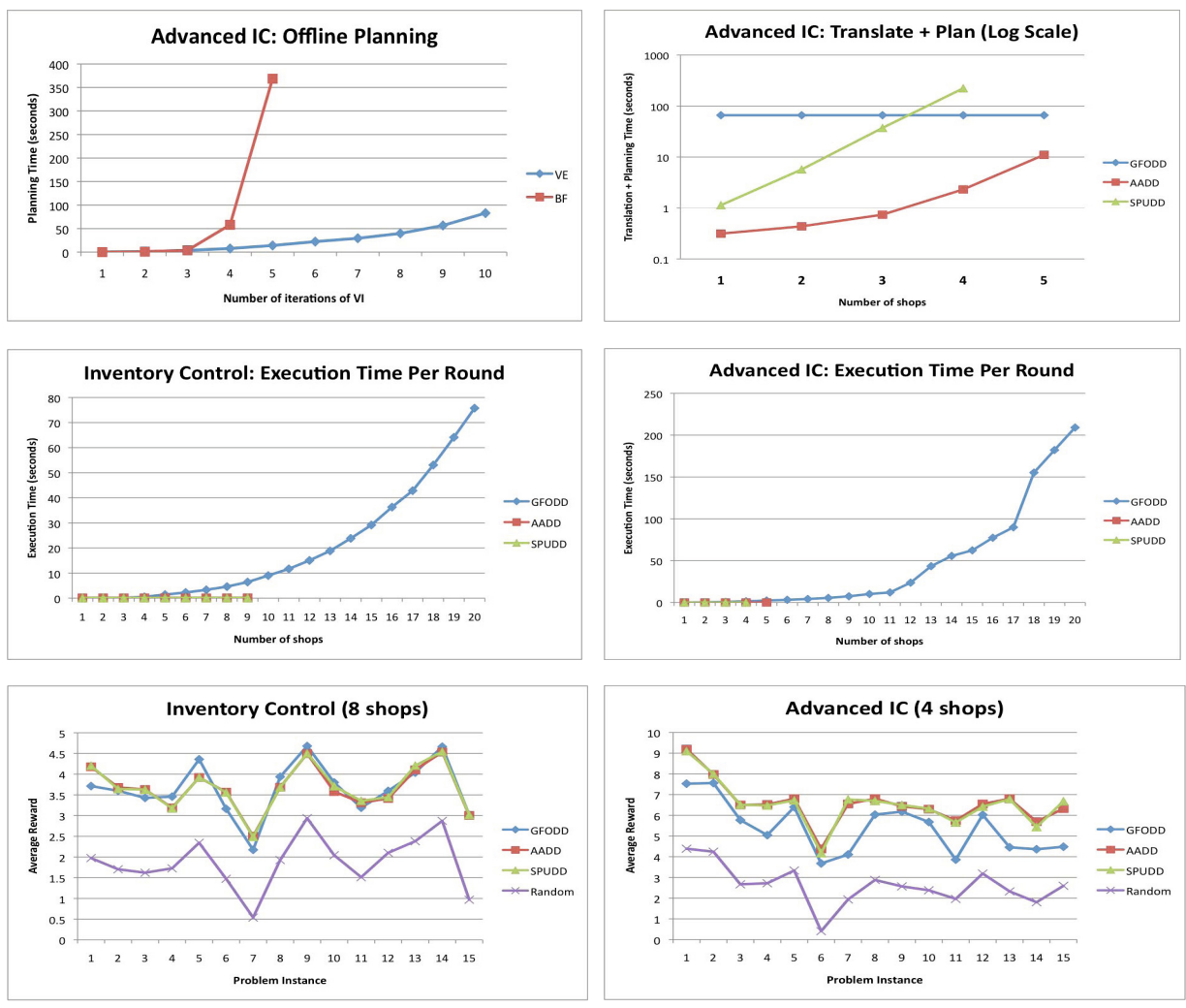

Fig. 4. Experimental Results

propositional descriptions using software provided for the IPPC 2011 planning competition [16]. All experiments were run on an Intel Core 2 Quad CPU @ 2.83GHz. Our system was given $3.5 \mathrm{~Gb}$ of memory and SPUDD and MADCAP were given $4 \mathrm{~Gb}$.

We tested all three systems on the IC domain as described above where shops and trucks have binary inventory levels (empty or full). We present results for the IC domain, because it satisfies all our assumptions and because the propositional systems fare better in this case. We also present results for a more complex IC domain (advanced IC or AIC below) where the inventory can be in one of 3 levels 0,1 and 2 and a shop can have one of 2 consumption rates 0.3 and 0.4. AIC does not satisfy assumption $\mathbf{A 3}$. As the experiments show, even with this small extension, the combinatorics render the propositional approach infeasible. In both cases, we constructed the set of focus states to include all possible states over 2 shops. This provides exact reduction for states with 2 shops but the reduction is approximate for larger states as in our experiments.

Figure 4 summarizes our results, which we discuss from left to right and top to bottom. The top left plot shows runtime as a function of iterations for AIC and illustrates that the variable elimination method is significantly faster than brute force evaluation and that it enables us to run many more iterations. The top right plot shows the total time (translation from RDDL to a propositional description and off-line planning for 10 iterations of VI) for the 3 systems for one problem instance per size for AIC. SPUDD 
runs out of memory and fails on more than 4 shops and MADCAP can handle at most 5 shops. Our planning time (being domain size agnostic) is constant. Runtime plots for IC are omitted but they show a similar qualitative picture, where the propositional systems fail with more than 8 shops for SPUDD and 9 shops for MADCAP.

The middle two plots show the cost of using the policies, that is, the on-line execution time as a function of increasing domain size in test instances. To control run time for our policies we show the time for the GFODD policy produced after 4 iterations, which is sufficient to solve any problem in IC and AIC 1 On-line time for propositional systems is fast for the domain sizes they solve, but our system can solve problems of much larger size (recall that the state space grows exponentially with the number of shops). The bottom two plots show the total discounted reward accumulated by each system (as well as a random policy) on 15 randomly generated problem instances averaged over 30 runs. In both cases all algorithms are significantly better than the random policy. In IC our approximate policy is not distinguishable from the optimal (SPUDD). In AIC the propositional policies are slightly better (differences are statistically significant). In summary, our system provides a non-trivial approximate policy but is sub-optimal in some cases, especially in AIC where $\mathbf{A 3}$ is violated. On the other hand its offline planning time is independent of domain size, and it can solve instances that cannot be solved by the propositional systems.

\section{Conclusions}

The paper presents service domains as an abstraction of planning problems with additive rewards and with multiple simultaneous but independent exogenous events. We provide a new relational SDP algorithm and the first complete analysis of such an algorithm with provable guarantees. In particular our algorithm, the template method, is guaranteed to provide a monotonic lower bound on the true value function under some technical conditions. We have also shown that this lower bound lies between the value of straight line plans and the true value function. As a second contribution we introduce new evaluation and reduction algorithms for the GFODD representation, that in turn facilitate efficient implementation of the SDP algorithm. Preliminary experiments demonstrate the viability of our approach and that our algorithm can be applied even in situations that violate some of the assumptions used in the analysis. The paper provides a first step toward analysis and solutions of general problems with exogenous events by focusing on a well defined subset of such models. Identifying more general conditions for existence of compact solutions, representations for such solutions, and associated algorithms is an important challenge for future work. In addition, the problems involved in evaluation and application of diagrams are computationally demanding. Techniques to speed up these computations are an important challenge for future work.

Acknowledgements. This work was partly supported by NSF under grants IIS-0964457 and IIS-0964705 and the CI fellows award for Saket Joshi. Most of this work was done when Saket Joshi was at Oregon State University.

\footnotetext{
${ }^{1}$ Our system does not achieve structural convergence because the reductions are not comprehensive. We give results at 4 iterations as this is sufficient for solving all problems in this domain. With more iterations, our policies are larger and their execution is slower.
} 


\section{References}

1. Bahar, R., Frohm, E., Gaona, C., Hachtel, G., Macii, E., Pardo, A., Somenzi, F.: Algebraic decision diagrams and their applications. In: Proceedings of the IEEE/ACM International Conference on Computer-Aided Design, pp. 188-191 (1993)

2. Boutilier, C., Dean, T., Hanks, S.: Decision-theoretic planning: Structural assumptions and computational leverage. Journal of Artificial Intelligence Research 11, 1-94 (1999)

3. Boutilier, C., Reiter, R., Price, B.: Symbolic dynamic programming for first-order MDPs. In: Proceedings of the International Joint Conference of Artificial Intelligence, pp. 690-700 (2001)

4. Guestrin, C., Koller, D., Parr, R., Venkataraman, S.: Efficient solution algorithms for factored MDPs. Journal of Artificial Intelligence Research 19, 399-468 (2003)

5. Hoey, J., St-Aubin, R., Hu, A., Boutilier, C.: SPUDD: Stochastic planning using decision diagrams. In: Proceedings of Uncertainty in Artificial Intelligence, pp. 279-288 (1999)

6. Hölldobler, S., Karabaev, E., Skvortsova, O.: FluCaP: a heuristic search planner for firstorder MDPs. Journal of Artificial Intelligence Research 27, 419-439 (2006)

7. Joshi, S., Kersting, K., Khardon, R.: Self-Taught decision theoretic planning with first-order decision diagrams. In: Proceedings of the International Conference on Automated Planning and Scheduling, pp. 89-96 (2010)

8. Joshi, S., Kersting, K., Khardon, R.: Decision theoretic planning with generalized first order decision diagrams. Artificial Intelligence 175, 2198-2222 (2011)

9. Joshi, S., Khardon, R.: Probabilistic relational planning with first-order decision diagrams. Journal of Artificial Intelligence Research 41, 231-266 (2011)

10. Joshi, S., Khardon, R., Tadepalli, P., Raghavan, A., Fern, A.: Solving relational MDPs with exogenous events and additive rewards. CoRR abs/1306 6302 (2013), http: //arxiv.org/abs/1306.6302

11. Kersting, K., van Otterlo, M., De Raedt, L.: Bellman goes relational. In: Proceedings of the International Conference on Machine Learning, pp. 465-472 (2004)

12. McMahan, H.B., Likhachev, M., Gordon, G.J.: Bounded real-time dynamic programming: RTDP with monotone upper bounds and performance guarantees. In: Proceedings of the International Conference on Machine Learning, pp. 569-576 (2005)

13. Puterman, M.L.: Markov Decision Processes: Discrete Stochastic Dynamic Programming. Wiley (1994)

14. Russell, S., Norvig, P.: Artificial Intelligence: A Modern Approach. Prentice Hall Series in Artificial Intelligence (2002)

15. Sanner, S.: First-order decision-theoretic planning in structured relational environments. Ph.D. thesis, University of Toronto (2008)

16. Sanner, S.: Relational dynamic influence diagram language (RDDL): Language description (2010), http: / / users . cecs . anu . edu . au/ sanner/IPPC2011/RDDL . pdf

17. Sanner, S., Boutilier, C.: Approximate solution techniques for factored first-order MDPs. In: Proceedings of the International Conference on Automated Planning and Scheduling, pp. 288-295 (2007)

18. Sanner, S., Boutilier, C.: Practical solution techniques for first-order MDPs. Artificial Intelligence 173, 748-788 (2009)

19. Sanner, S., Uther, W., Delgado, K.: Approximate dynamic programming with affine ADDs. In: Proceeding of the International Conference on Autonomous Agents and Multiagent Systems, pp. 1349-1356 (2010)

20. Wang, C., Joshi, S., Khardon, R.: First-Order decision diagrams for relational MDPs. Journal of Artificial Intelligence Research 31, 431-472 (2008) 